\title{
With Wronskian through the Looking Glass
}

\author{
Vassily GORBOUNOV ${ }^{\dagger}$ and Vadim SCHECHTMAN ${ }^{\S}$ \\ $\dagger$ HSE University, Russia \\ ¥ Laboratory of Algebraic Geometry and Homological Algebra, \\ Moscow Institute of Physics and Technology, Dolgoprudny, Russia \\ E-mail:vgorb10@gmail.com \\ $\S$ Institut de Mathématiques de Toulouse, Université Paul Sabatier, \\ 118 Route de Narbonne, 31062 Toulouse, France \\ E-mail: schechtman@math.ups-tlse.fr
}

Received September 01, 2020, in final form December 27, 2020; Published online January 02, 2021 https://doi.org/10.3842/SIGMA.2021.001

\begin{abstract}
In the work of Mukhin and Varchenko from 2002 there was introduced a Wronskian map from the variety of full flags in a finite dimensional vector space into a product of projective spaces. We establish a precise relationship between this map and the Plücker map. This allows us to recover the result of Varchenko and Wright saying that the polynomials appearing in the image of the Wronsky map are the initial values of the tau-functions for the Kadomtsev-Petviashvili hierarchy.
\end{abstract}

Key words: MKP hierarchies; critical points; tau-function; Wronskian

2020 Mathematics Subject Classification: 37K20; 81R10; 35C08

To Vitaly Tarasov and Alexander Varchenko, as a token of friendship

Всё смешалось в доме Облонских

\section{Introduction}

Let $G=\mathrm{GL}_{n}(\mathbb{C}), T \subset B_{-} \subset G$ the subgroups of diagonal and lower triangular matrices, $X=B_{-} \backslash G$ the variety of full flags in $V=\mathbb{C}^{n}$.

We have the Plücker embedding

$$
\mathcal{P} \ell=\left(\mathcal{P} \ell_{1}, \ldots, \mathcal{P} \ell_{n-1}\right): X \hookrightarrow \mathbb{P}:=\prod_{i=1}^{n-1} \mathbb{P}^{C_{n}^{i}-1},
$$

where $C_{n}^{i}=\left(\begin{array}{c}n \\ i\end{array}\right)$. On the other hand, in [9] there was introduced a map

$$
\mathfrak{W}: \quad X \longrightarrow\left(\mathbb{P}^{N}\right)^{n-1}
$$

( $N$ being big enough), which we call the Wronskian map since its definition uses a lot of Wronskians. This map has been studied in [10]. We will see below that $\mathfrak{W}$ lands in a subspace

$$
\prod_{i=1}^{n-1} \mathbb{P}^{i(n-i)-1} \subset\left(\mathbb{P}^{N}\right)^{n-1},
$$

This paper is a contribution to the Special Issue on Representation Theory and Integrable Systems in honor of Vitaly Tarasov on the 60th birthday and Alexander Varchenko on the 70th birthday. The full collection is available at https://www.emis.de/journals/SIGMA/Tarasov-Varchenko.html 
so we will consider it as a map

$$
\mathfrak{W}=\left(\mathfrak{W}_{1}, \ldots, \mathfrak{W}_{n-1}\right): \quad X \longrightarrow \mathbb{P}^{\prime}:=\prod_{i=1}^{n-1} \mathbb{P}^{i(n-i)-1} .
$$

The present note, which may be regarded as a postscript to [10], contains some elementary remarks on the relationship between $\mathcal{P} \ell$ and $\mathfrak{W}$.

We define for each $1 \leq i \leq n-1$ a linear contraction map

$$
c_{i}: \mathbb{P}^{C_{n}^{i}-1} \longrightarrow \mathbb{P}^{i(n-i)-1},
$$

such that

$$
\mathfrak{W}_{i}=c_{i} \circ \mathcal{P} \ell_{i},
$$

see Theorem 3.3.

For $g \in G$ let $\bar{g} \in X$ denote its image in $X$; let

$$
\mathfrak{W}_{i}(g)=\left(a_{0}(g): \cdots: a_{i(n-i)}(g)\right),
$$

and consider a polynomial

$$
y_{i}(g)(x)=\sum_{q=0}^{i(n-i)-1} a_{q}(g) \frac{x^{q}}{q !} .
$$

As a corollary of Theorem 3.3 we deduce that the polynomials $y_{i}(g)$ are nothing else but the initial values of the tau-functions for the KP hierarchy, see Theorems 4.8 and 4.14; this assertion is essentially [12, Lemma 5.7].

As another remark we reinterpret in Section 5 the $W 5$ identity instrumental in [10] as a particular case of the classical Desnanot-Jacobi formula, and explain its relation to Wronskian mutations studied in $[9,10]$. We note some interesting related references $[4,6]$.

\section{Wronsky map}

We fix a base commutative ring $\mathbf{k} \supset \mathbb{Q}$. Let

$$
\mathfrak{f}=\left(f_{1}(t), \ldots, f_{n}(t)\right)
$$

be a sequence of rational functions $f_{i}(t) \in \mathbf{k}(t)$. Its Wronskian matrix is by definition an $n \times n$ matrix

$$
\mathcal{W}(\mathfrak{f})=\left(f_{i}^{(j)}(t)\right),
$$

where $f^{(j)}(t)$ denotes the $j$-th derivative.

The determinant of $\mathcal{W}(\mathfrak{f})$ is the Wronskian of $\mathfrak{f}$ :

$$
W(\mathfrak{f})=\operatorname{det}(\mathcal{W}(\mathfrak{f})) .
$$

If $A=\left(a_{i j}\right) \in \mathfrak{g l}_{n}(\mathbf{k})$ is a scalar matrix,

$$
\begin{aligned}
& W(\mathfrak{f} A)=\operatorname{det}(A) W(\mathfrak{f}), \\
& W\left(f_{1}, \ldots, f_{n}\right)^{\prime}=\sum_{i=1}^{n} W\left(f_{1}, \ldots, f_{i}^{\prime}, \ldots, f_{n}\right) .
\end{aligned}
$$




\section{$2.1 \quad$ The map $\mathfrak{W}$}

Let

$$
M=\left(b_{i j}\right)_{1 \leq i \leq n, 1 \leq j \leq m} \in \operatorname{Mat}_{n, m}(\mathbf{k})
$$

be a rectangular matrix. Let us associate to it a sequence of polynomials of degree $m-1$

$$
\mathfrak{b}(M)=\left(b_{1}(M, t), \ldots, b_{n}(M, t)\right), \quad b_{i}(t)=\sum_{j=0}^{m-1} b_{i, j+1} \frac{t^{j}}{j !} .
$$

In general we identify the space of polynomials of degree $\leq m-1$ with the space of $\mathbf{k}$-points of an affine space:

$$
\mathbf{k}[t]_{\leq m-1} \stackrel{\sim}{\longrightarrow} \mathbb{A}^{m+1}(\mathbf{k}), \quad \sum_{j=0}^{m-1} b_{j} \frac{t^{j}}{j !} \mapsto\left(b_{0}, \ldots, b_{m-1}\right) .
$$

For $1 \leq j \leq n$ let $\mathfrak{b}_{\leq j}(M)$ denote the truncated sequence

$$
\mathfrak{b}(M)_{\leq j}=\left(b_{1}(M, t), \ldots, b_{j}(M, t)\right) .
$$

We define a sequence of polynomials

$$
\mathfrak{W}(M)=\left(y_{1}(M), \ldots, y_{n-1}(M)\right):=\left(W\left(\mathfrak{b}(M)_{\leq 1}\right), \ldots, W\left(\mathfrak{b}(M)_{\leq n-1}\right)\right) \in \mathbf{k}[t]^{n-1} .
$$

Note that if $n=m$ then

$$
W(\mathfrak{b}(M))=\operatorname{det} M,
$$

it is a constant polynomial.

If $A \in \mathfrak{g l}_{n}(k)$ then

$$
\mathfrak{b}(A M)=\mathfrak{b}(M) A^{t}
$$

It follows that if $e_{i j}(a), i>j$, is a lower triangular elementary matrix then

$$
\mathfrak{b}\left(e_{i j}(a) M\right)=\left(b_{1}(M), \ldots, b_{j}(M)+a b_{i}(M), b_{j+1}(M), \ldots\right),
$$

whence

$$
\left.\mathfrak{W}\left(e_{i j}(a) M\right)\right)=\mathfrak{W}(M) .
$$

It follows that for any $A \in N_{-}(\mathbf{k})$ (a lower triangular with 1's on the diagonal)

$$
\mathfrak{W}(A M)=\mathfrak{W}(M) .
$$

On the other hand, if

$$
D=\operatorname{diag}\left(d_{1}, \ldots, d_{n}\right) \in \mathfrak{g l}_{n}(\mathbf{k})
$$

then

$$
\mathfrak{W}(D M)=\prod_{i=1}^{n} d_{i} \cdot \mathfrak{W}(M) .
$$

In other words, if $A \in B_{-}(n, \mathbf{k})$ (the lower Borel),

$$
\mathfrak{W}(A M)=\operatorname{det}(A) \mathfrak{W}(M),
$$

which of course is seen immediately. 


\subsection{Degrees and Bruhat decomposition}

Suppose that $n=m$. We will denote by $G=\mathrm{GL}_{n}, B_{-} \subset G$ the lower triangular Borel, $N_{-} \subset B_{-}$ etc.

For a matrix $g \in G(\mathbf{k})$ let $\mathfrak{W}(g)=\left(y_{1}(g), \ldots, y_{n-1}(g)\right)$, and consider the vector of degrees

$$
d(g)=\left(d_{1}(g), \ldots d_{n-1}(g)\right)=\left(\operatorname{deg} y_{1}(g), \ldots, \operatorname{deg} y_{n-1}(g)\right) \in \mathbb{N}^{n-1} .
$$

It turns out that $d(g)$ can take only $n$ ! possible values situated in vertices of a permutohedron.

Namely, consider the Bruhat decomposition

$$
G(\mathbf{k})=\cup_{w \in W} B_{-}(\mathbf{k}) w B_{-}(\mathbf{k}),
$$

$W=S_{n-1}=W(G, T)$ being the Weyl group.

Identify $\mathbb{N}^{n-1}$ with the root lattice $Q$ of $G^{s}:=\mathrm{SL}_{n}$ using the standard base $\left\{\alpha_{1}, \ldots, \alpha_{n-1}\right\} \subset Q$ of simple roots.

Then it follows from [9, Theorem 3.12], that for $g \in B(w):=B_{-}(\mathbf{k}) w B_{-}(\mathbf{k})$

$$
d(g)=w * \mathbf{0},
$$

where $\mathbf{0}=(0, \ldots, 0)$ and $*$ denotes the usual shifted Weyl group action

$$
w * \alpha=w(\alpha-\rho)+\rho .
$$

In other words, if

$$
w * \mathbf{0}=\sum_{i=1}^{n-1} d_{i}(w) \alpha_{i}
$$

then

$$
d_{i}(g)=d_{i}(w), \quad 1 \leq i \leq n-1 .
$$

Example 2.1. Let $n=3$. For $g=\left(a_{i j}\right) \in \mathrm{GL}_{3}(\mathbf{k})$

$$
\begin{aligned}
& y_{1}(g)=a_{11}+a_{12} x+a_{13} \frac{x^{2}}{2}, \\
& y_{2}(g)=\Delta_{11}(g)+\Delta_{13}(g) x+\Delta_{23}(g) \frac{x^{2}}{2} .
\end{aligned}
$$

Here $\Delta_{i j}(g)$ denotes the $2 \times 2$ minor of $g$ picking the first two rows and $i$-th and $j$-th columns.

We have two simple roots $\alpha_{1}, \alpha_{2}$.

For $g \in \mathrm{GL}_{3}(\mathbf{k})$ the vector $d(g)$ can take 6 possible values: $(0,0),(1,0),(0,1),(1,2),(2,1)$, and $(2,2)$, these vectors forming a hexagon, cf. [9, Section 3.5].

One checks directly that

$$
B_{-}=d^{-1}(0,0) \text {. }
$$

This means that $g \in B_{-}$if and only if $a_{12}=a_{13}=\Delta_{23}(g)=0$.

Similarly

$$
B_{-}(12) B_{-}=d^{-1}(1,0), \quad B_{-}(23) B_{-}=d^{-1}(0,1), \quad B_{-}(123) B_{-}=d^{-1}(2,1),
$$

i.e., $g \in B_{-}(123) B_{-}$if and only if $\Delta_{23}(g)=0$;

$$
B_{-}(132) B_{-}=d^{-1}(2,1), \quad B_{-}(13) B_{-}=d^{-1}(2,2)
$$

(the big cell). This means that $g \in B_{-}(13) B_{-}$if and only if $a_{12} \neq 0, a_{13} \neq 0, \Delta_{23}(g) \neq 0$.

These formulas may be understood as a criterion for recognizing the Bruhat cells in $\mathrm{GL}_{3}$, cf. [5]. 
Therefore the Wronskian map induces maps

$$
\mathfrak{W}(w): \quad B(w) \longrightarrow \prod_{i=1}^{n-1} \mathbb{P}^{d_{i}(w)}
$$

for each $w \in W$.

\subsection{Induced map on the flag space}

Let $D \in \mathbb{N}$ be such that $d_{i}(g) \leq D$ for all $g \in G, i \in[n-1]:=\{1, \ldots, n-1\}$.

The invariance (2.1) implies that $\mathfrak{W}$ induces a map from the base affine space

$$
\mathfrak{W}_{\tilde{\mathcal{F}} \ell_{-}}: \tilde{\mathcal{F}} \ell_{-}:=N_{-} \backslash G \longrightarrow \mathbf{k}[t]_{\leq D}^{n-1} \cong\left(\mathbb{A}^{D+1}\right)^{n-1}(\mathbf{k}),
$$

while (2.2) implies that $\mathfrak{W}$ induces a map from the full flag space

$$
\mathfrak{W}_{\mathcal{F} \ell_{-}}: \quad \mathcal{F} \ell_{-}:=B_{-} \backslash G \longrightarrow \mathbb{P}(\mathbf{k}[t])_{\leq D}^{n-1} \cong\left(\mathbb{P}^{D}\right)^{n-1}(\mathbf{k}) .
$$

More explicitly: we can assign to an arbitrary matrix $g=\left(b_{i j}\right) \in G$ a flag in $V=\mathbf{k}^{n}$

$$
F(g)=V_{1}(g) \subset \cdots \subset V_{n}(g)=V,
$$

whose $i$-th space $V_{i}(g)$ is spanned by the first $i$ row vectors of $g$

$$
v_{j}(g)=\left(b_{j 1}, \ldots, b_{j n}\right) \in V, \quad 1 \leq j \leq i .
$$

It is clear that $F(g)=F(n g)$ for $n \in B_{-}$, and the map

$$
F: G \longrightarrow \mathcal{F} \ell(V),
$$

where $\mathcal{F} \ell(V)$ is the space of full flags in $V$, induces an isomorphism

$$
B_{-} \backslash G \stackrel{\sim}{\longrightarrow} \mathcal{F} \ell(V) .
$$

On the other hand consider the restriction of $F$ to the upper triangular group

$$
F_{N}: N \hookrightarrow \mathcal{F} \ell(V)
$$

this map is injective and its image is the big Schubert cell.

We may also consider the composition

$$
\mathfrak{W}_{N}: N \hookrightarrow \mathcal{F} \ell_{-} \stackrel{\mathfrak{W}_{\mathcal{F} \ell_{-}}}{\longrightarrow}\left(\mathbb{P}^{D}\right)^{n-1} .
$$

We will see below (cf. Section 3.6) that this map is an embedding.

\subsection{Partial flags}

More generally, for any unordered partition

$$
\lambda: n=n_{1}+\cdots+n_{p}, \quad n_{i} \in \mathbb{Z}_{\mathbb{Z}>0}
$$

we define in a similar way a map

$$
\mathfrak{W}: \quad \mathcal{F} \ell_{\lambda,-}:=P_{\lambda,-} \backslash G \longrightarrow\left(\mathbb{P}^{D}\right)^{p-1} .
$$

For example, for $p=2$ (Grassmanian case) corresponding to a partition $\lambda=i+(n-i)$

$$
\mathfrak{W}: \quad \mathcal{F} \ell_{\lambda,-}=\mathrm{Gr}_{n}^{i} \longrightarrow \mathbb{P}^{D}
$$




\section{Coefficients of Wronskians}

\subsection{Plücker map}

\subsubsection{Schubert cells in a Grassmanian}

For $i \in[n]:=\{1, \ldots, n\}$ let $\mathcal{C}_{n}^{i}$ denote the set of all $i$-element subsets of $[n]$.

The natural action of $W=S_{n}$ on $\mathcal{C}_{n}^{i}$ identifies

$$
\mathcal{C}_{n}^{i} \cong S_{n} / S_{i}
$$

Let $P_{i} \subset G=\mathrm{GL}_{n}$ denote the stabilizer of the coordinate subspace $\mathbb{A}^{i} \subset \mathbb{A}^{n}$, so that

$$
G / P_{i} \cong \mathrm{Gr}_{n}^{i},
$$

the Grassmanian of $i$-planes in $\mathbb{A}^{n}$. The Bruhat lemma gives rise to an isomorphism

$$
\mathcal{C}_{n}^{i} \cong B \backslash G / P_{i} .
$$

This set may also be interpreted as "the set of $\mathbb{F}_{1}$-points"

$$
\mathcal{C}_{n}^{i}=\operatorname{Gr}_{n}^{i}\left(\mathbb{F}_{1}\right),
$$

whose cardinality is a binomial coefficient

$$
\left|\mathcal{C}_{n}^{i}\right|=C_{n}^{i}=\left(\begin{array}{c}
n \\
i
\end{array}\right)
$$

\subsubsection{A Plücker map}

Consider a matrix

$$
M=\left(b_{i j}\right)_{i \in[n], j \in[m]} \in \operatorname{Mat}_{n, m}(\mathbf{k})
$$

with $n \leq m$. For any $j \in[n] M_{\leq j}$ will denote the truncated matrix

$$
M_{\leq j}=\left(b_{i p}\right)_{i \in[j], p \in[m]} \in \operatorname{Mat}_{j, m}(\mathbf{k}) .
$$

We suppose that $\operatorname{rank}(M)=n$.

For any $j \in[n]$ consider the set of $j \times j$ minors of $M$

$$
p_{j}(M)=\left(\Delta_{[j], I}\right)_{I \in \mathcal{C}_{m}^{j}} \in \mathbb{A}^{C_{m}^{j}}(\mathbf{k})
$$

or the same set up to a multiplication by a scalar

$$
\bar{p}_{j}(M)=\pi\left(p_{j}(M)\right)=\mathbb{P}^{C_{m}^{j}-1}(\mathbf{k}),
$$

where $\pi: \mathbb{A}^{C_{m}^{j}}(\mathbf{k}) \backslash\{\mathbf{0}\} \longrightarrow \mathbb{P}^{C_{m}^{j}-1}(\mathbf{k})$ is the canonical projection.

We will use notations

$$
\tilde{\mathcal{P}} \ell(M)=\left(p_{1}(M), \ldots, p_{m}(M)\right) \in \prod_{j=1}^{m} \mathbb{A}_{m}^{C_{m}^{j}}(\mathbf{k})
$$

and

$$
\mathcal{P} \ell(M)=\left(\bar{p}_{1}(M), \ldots, \bar{p}_{m}(M)\right) \in \prod_{j=1}^{m} \mathbb{P}^{C_{m}^{j}-1}(\mathbf{k}) .
$$


Suppose that $m=n$, so we get maps

$$
\tilde{\mathcal{P} \ell}=\left(\mathcal{P} \ell_{1}, \ldots, \mathcal{P} \ell_{n}\right): \mathrm{GL}_{n} \longrightarrow \prod_{j=1}^{n} \mathbb{A}^{C_{n}^{j}}
$$

and

$$
\mathcal{P} \ell: \quad \mathrm{GL}_{n} \longrightarrow \prod_{j=1}^{n} \mathbb{P}^{C_{n}^{j}-1} .
$$

It is clear that $\mathcal{P} \ell(z M)=\mathcal{P} \ell(M)$ for $z \in N_{-} \subset G=\mathrm{GL}_{n}$ and $\mathcal{P} \ell(b M)=\mathcal{P} \ell(M)$ for $b \in B_{-} \subset G$, so $\tilde{\mathcal{P}} \ell, \mathcal{P} \ell$ induce maps

$$
\mathcal{P} \ell_{\tilde{\mathcal{F}} \ell}: \tilde{\mathcal{F}} \ell_{-}=N_{-} \backslash G \longrightarrow \prod_{j=1}^{n} \mathbb{A}^{C_{n}^{j}}
$$

and

$$
\mathcal{P} \ell_{\mathcal{F} \ell}: \quad \mathcal{F} \ell_{-}=B_{-} \backslash G \longrightarrow \prod_{j=1}^{n} \mathbb{P}^{C_{n}^{j}-1} .
$$

\subsection{Schubert cells in Grassmanians}

For $i \in[n]$ consider the $i$-th Plücker map

$$
\mathcal{P} \ell_{i}: G \longrightarrow \mathbb{A}^{C_{n}^{i}} \text {. }
$$

We will compare it with the $i$-th component of the Wronskian map

$$
\mathfrak{W}_{i}: G \longrightarrow \mathbb{A}^{d_{i}+1}, \quad g \mapsto y_{i}(g) .
$$

To formulate the result we will use the Schubert decomposition from Section 3.1.1.

Let $p=\ell(w)$ denote the length of a minimal decomposition

$$
w=s_{j_{1}} \cdots s_{j_{p}}
$$

into a product of Coxeter generators $s_{j}=(j, j+1)$.

Let

$$
I_{0}=I_{\min }=\{1, \ldots, i\} \in \mathcal{C}_{n}^{i} .
$$

Sometimes it is convenient to depict elements of $\mathcal{C}_{n}^{i}$ as sequences

$$
I=\left(e_{1} \ldots e_{n}\right), \quad e_{j} \in\{0,1\}, \quad \sum e_{j}=i .
$$

In this notation

$$
I_{0}=1 \ldots 10 \ldots 0 \text {. }
$$

We define a length map

$$
\mathfrak{l}: \mathcal{C}_{n}^{i} \longrightarrow \mathbb{Z}_{\geq 0}
$$

as follows: identify $\mathcal{C}_{n}^{i} \cong S_{n} / S_{i}$, then for $\bar{x} \in \mathcal{C}_{n}^{i} \ell(\bar{x})$ is the minimal length of a representative $x \in S_{n}$. 
Example 3.1. $\mathfrak{l}\left(I_{0}\right)=0$. The element of maximal length is

$$
I_{\max }=0 \ldots 01 \ldots 1 \text {. }
$$

Its length is

$$
\mathfrak{l}\left(I_{\max }\right)=i(n-i) .
$$

Claim 3.2. Let $w_{0} \in S_{n}=W\left(\mathrm{GL}_{n}\right)$ denote the element of maximal length. Then

$$
d_{i}:=d\left(w_{0}\right)=\mathfrak{l}\left(I_{\max }\right)=i(n-i) .
$$

This is a particular case of a more general statement, see below Corollary 3.6.

For each $j \geq 0$ consider the subset

$$
\mathcal{C}_{n}^{i}(j)=\mathfrak{l}^{-1}(j) \subset \mathcal{C}_{n}^{i},
$$

so that

$$
\mathcal{C}_{n}^{i}=\coprod_{j=0}^{d_{i}} \mathcal{C}_{n}^{i}(j)
$$

For example

$$
\mathcal{C}_{n}^{i}(0)=\left\{I_{0}\right\}, \quad \mathcal{C}_{n}^{i}\left(d_{i}\right)=\left\{I_{\max }\right\} .
$$

\subsubsection{Symmetry}

$$
\left|\mathcal{C}_{n}^{i}(j)\right|=\left|\mathcal{C}_{n}^{i}\left(d_{i}-j\right)\right|
$$

\subsubsection{Range}

$\mathcal{C}_{n}^{i}(j) \neq \varnothing$ iff $0 \leq j \leq d_{i}$. In other words, the range of $\mathfrak{l}$ is $\left\{0, \ldots, d_{i}\right\}$.

The following statement is the main result of the present note.

\subsection{From $\mathcal{P} \ell$ to $\mathfrak{W}:$ a contraction}

\section{Theorem 3.3.}

$$
y_{i}(g)(t)=\sum_{I \in \mathcal{C}_{n}^{i}} \Delta_{[i], I}(g) m(I) \frac{t^{\mathfrak{l}(I)}}{\mathfrak{l}(I) !}=\sum_{j=0}^{d_{i}}\left(\sum_{I \in \mathcal{C}_{n}^{i}(j)} m(I) \Delta_{[i], I}(g)\right) \frac{t^{j}}{j !},
$$

where the numbers $m(I) \in \mathbb{Z}_{>0}$ are defined below, see Section 3.4.

In other words, the map $\mathfrak{l}$ induces a contraction map

$$
\begin{aligned}
& c=c_{i}: \mathbb{A}^{\mathcal{C}_{n}^{i}} \longrightarrow \mathbf{k}[t]_{\leq d_{i}}, \\
& c\left(\left(a_{I}\right)_{I \in \mathcal{C}_{n}^{i}}\right)=\sum_{j=0}^{d_{i}}\left(\sum_{I \in \mathfrak{I}^{-1}(j)} m(I) a_{I}\right) \frac{t^{j}}{j !} .
\end{aligned}
$$

Then

$$
\mathfrak{W}_{i}=c_{i} \circ \mathcal{P} \ell_{i} .
$$


We will also use the reciprocal polynomials

$$
\tilde{y}_{i}(g)(x)=x^{d_{i}} y_{i}(g)\left(x^{-1}\right) .
$$

Proof of Theorem 3.3 is given below after some preparation.

\section{Examples 3.4.}

(i) Let $n=4, i=2$. A formula for $y_{2}(g), g \in N_{4}$ is given in [10, equation (5.11)]:

$$
y_{2}(g)(t)=\Delta_{12}(g)+\Delta_{13}(g) t+\left(\Delta_{14}(g)+\Delta_{23}(g)\right) \frac{t^{2}}{2}+2 \Delta_{24}(g) \frac{t^{3}}{6}+2 \Delta_{34}(g) \frac{t^{4}}{24},
$$

where for brevity

$$
\Delta_{I}:=\Delta_{12, I}
$$

for $I \subset[4]$.

(ii) More generally, for $g \in \mathrm{GL}_{n}$

$$
\begin{aligned}
y_{2}(g)= & \Delta_{12}(g)+\Delta_{13}(g) t+\left(\Delta_{14}(g)+\Delta_{23}(g)\right) \frac{t^{2}}{2} \\
& +\left(2 \Delta_{24}(g)+\Delta_{15}(g)\right) \frac{t^{3}}{6}+\left(2 \Delta_{34}(g)+2 \Delta_{25}(g)+\Delta_{16}(g)\right) \frac{t^{4}}{24}+\cdots,
\end{aligned}
$$

$\operatorname{deg} y_{2}(g) \leq n(n-2)$, the exact degree depends on the Bruhat cell which $g$ belongs to.

(iii) Let again $n=4$. Then (see [10, equation (5.11)])

$$
y_{3}(g)=\Delta_{123}(g)+\Delta_{124}(g) t+\Delta_{134}(g) \frac{t^{2}}{2}+\Delta_{234}(g) \frac{t^{3}}{6} .
$$

\subsection{Creation operators $\Delta_{i}$}

Fix $n \geq 2$. For

$$
I=\left\{i_{1}, \ldots, i_{k}\right\} \in \mathcal{C}_{n}^{k}
$$

we imply that $i_{1}<\cdots<i_{k}$.

We call $i=i_{p}$ admissible if either $p=k$ and $i_{p}<n$ or $i_{p+1}>i_{p}+1$. We denote by $I^{o} \subset I$ the subset of admissible elements.

For each $i=i_{p} \in I^{o}$ we define a new set

$$
\Delta_{i} I=\left\{i_{1}^{\prime}, \ldots, i_{k}^{\prime}\right\}
$$

where $i_{q}^{\prime}=i_{q}$ if $q \neq p$, and $i_{p}^{\prime}=i_{p}+1$.

The reader should compare this definition with operators defining a representation of the nil-Temperley-Lieb algebra from [2, equation (2.4.6)], cf. also [1] and references therein.

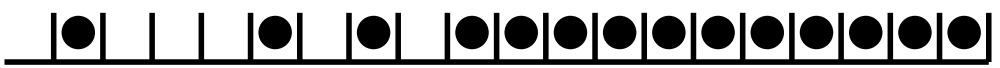

$$
-8-7-6-5-4-3-2-10 \quad 1
$$

Figure 1. "Balls in boxes" picture.

Recall the representation (3.1) of elements of $\mathcal{C}_{n}^{k}$ : where we imagine the 1 's as $k$ "balls" sitting in $n$ "boxes". An operation $\Delta_{i}$ means moving the ball in $i$-th box to the right, which is possible if the $(i+1)$-th box is free. 
Each $I \in \mathcal{C}_{n}^{k}$ may be written as

$$
I=\Delta_{j_{p}} \cdots \Delta_{j_{1}} I_{\min }
$$

for some $j_{1}, \ldots, j_{p}$. This is clear from the balls in boxes picture.

Let $\mathfrak{m}(I)$ denote the set of all sequences $j_{1}, \ldots, j_{p}$ such that (3.5) holds, and

$$
m(I):=|\mathfrak{m}(I)|
$$

Claim 3.5. The lengths $p$ of all sequences $\left(j_{1}, \ldots, j_{p}\right) \in \mathfrak{m}(I)$ are the same, namely $p=\mathfrak{l}(I)$. Here $\mathfrak{l}(I)$ is from (3.2).

Proof. Clear from balls in boxes picture.

Corollary 3.6. Let

$$
I=\left\{a_{1}, \ldots, a_{k}\right\}
$$

then

$$
\mathfrak{l}(I)=\sum_{i=1}^{k}\left(a_{i}-i\right) .
$$

To put it differently, define a graph $\Gamma_{n}^{k}$ whose set of vertices is $\mathcal{C}_{n}^{k}$, the edges having the form

$$
I \longrightarrow \Delta_{i} I
$$

(or otherwise define an obvious partial order on $\mathcal{C}_{n}^{i}$ ). Then $\mathfrak{m}(I)$ is the set of paths in $\Gamma_{n}^{k}$ going from the minimal element $[k]$ to $I$.

\subsubsection{Symmetry}

This graph can be turned upside down. Clear from the "balls in boxes" description.

\subsection{Generalized Wronskians and the derivative}

Let

$$
\mathfrak{f}=\left(f_{1}(t), f_{2}(t), \ldots\right)
$$

be a sequence of functions. We can assign to it a $\mathbb{Z}_{\geq 1} \times \mathbb{Z}_{\geq 1}$ Wronskian matrix

$$
\mathcal{W}(\mathfrak{f})=\left(f_{i}^{(j-1)}\right)_{i, j \geq 1} .
$$

For each $I=\left\{i_{1}, \ldots, i_{k}\right\} \in \mathcal{C}_{\infty}^{k}$ let $\mathcal{W}_{I}(\mathfrak{f})$ denote a $(k \times k)$-minor of $\mathcal{W}(\mathfrak{f})$ with rows $i_{1}, \ldots, i_{k}$ and columns $1,2, \ldots, k$, and let

$$
W_{I}(\mathfrak{f})=\operatorname{det} \mathcal{W}_{I}(\mathfrak{f}) .
$$

Lemma 3.7. The derivative

$$
W_{I}(\mathfrak{f})^{\prime}=\sum_{i \in I^{o}} W_{\Delta_{i} I}(\mathfrak{f}) .
$$




\section{Corollary 3.8.}

$$
W_{I}(\mathfrak{f})^{(p)}=\sum_{\left(i_{1}, \ldots, i_{p}\right) \text { composable }} W_{\Delta_{i_{p}} \cdots \Delta_{i_{1}} I}(\mathfrak{f}),
$$

where a sequence $\left(i_{1}, \ldots, i_{p}\right)$ is called composable if $i_{q} \in\left(\Delta_{i_{q-1}} \cdots \Delta_{i_{1}} I\right)^{o}$ for all $q$.

Proof of Theorem 3.3. We shall use a formula: if

$$
y(t)=\sum_{i \geq 0} a_{i} \frac{t^{i}}{i !}
$$

then

$$
a_{i}=y^{(i)}(0) .
$$

Let $g=\left(b_{i j}\right) \in \mathrm{GL}_{n}$,

$$
b_{i}(t)=\sum_{j=0}^{n-1} b_{i, j+1} \frac{t^{j}}{j !}, \quad 1 \leq i \leq n .
$$

By definition

$$
y_{i}(g)(t)=W\left(b_{1}(t), \ldots, b_{i}(t)\right), \quad 1 \leq i \leq n .
$$

For $I, J \subset[n]$ let $M_{I J}(g)$ denote the submatrix of $g$ lying on the intersection of the lines (columns) with numbers $i \in I(j \in J)$, so that

$$
\Delta_{I J}(g)=\operatorname{det} M_{I J}(g) .
$$

We see that the constant term

$$
y_{i}(g)(0)=\operatorname{det}\left(M_{[i],[i]}(g)^{t}\right)=\Delta_{[i]}(g),
$$

where $M^{t}$ denotes the transposed matrix.

To compute the other coefficients we use Lemma 3.7 and Corollary 3.8. So

$$
y_{i}^{\prime}(0)=\operatorname{det}\left(M_{[i], \Delta_{i}[i]}(g)^{t}\right)=\Delta_{[i], \Delta_{i}[i]}(g),
$$

and more generally

$$
\begin{aligned}
y_{i}^{(p)}(0) & =\sum_{\left(i_{1}, \ldots, i_{p}\right) \text { composing }} \operatorname{det}\left(M_{[i], \Delta_{i_{p}} \cdots \Delta_{i_{1}}[i]}(g)^{t}\right) \\
& =\sum_{\left(i_{1}, \ldots, i_{p}\right) \text { composing }} \Delta_{[i], \Delta_{i_{p}} \cdots \Delta_{i_{1}}[i]}(g),
\end{aligned}
$$

which implies the formula. 


\subsection{Triangular theorem}

Consider an upper triangular unipotent matrix $g \in N \subset \mathrm{GL}_{n}(\mathbf{k})$. We claim that $g$ may be reconstructed uniquely from the coefficients of polynomials $y_{1}(g), \ldots, y_{n-1}(g)$.

More precisely, to get the first $i$ rows of $g$ we need only a truncated part of the first $i$ polynomials

$$
\left(y_{1}(g)=y_{1}(g)_{\leq n-1}, y_{2}(g)_{\leq n-2}, \ldots, y_{i}(g)_{\leq i}\right) .
$$

This is the contents of [10, Theorem 5.3]. We explain how it follows from our Theorem 3.3.

To illustrate what is going on consider an example $n=5$. Let

$$
g=\left(\begin{array}{ccccc}
1 & a_{1} & a_{2} & a_{3} & a_{4} \\
0 & 1 & b_{2} & b_{3} & b_{4} \\
0 & 0 & 1 & c_{3} & c_{4} \\
0 & 0 & 0 & 1 & d_{4} \\
0 & 0 & 0 & 0 & 1
\end{array}\right)
$$

We have $y_{1}(g)=b_{1}(g)$, so we get the first row of $g$, i.e., the elements $a_{i}$, from $y_{1}(g)$.

Next,

$$
\begin{aligned}
y_{2}(g) & =\Delta_{12}(g)+\Delta_{13}(g) x+\left(\Delta_{14}(g)+\Delta_{23}(g)\right) \frac{x^{2}}{2}+\left(\Delta_{15}(g)+\cdots\right) \frac{x^{3}}{6}+\cdots \\
& =1+b_{2} x+\left(b_{3}+a_{1} b_{2}-a_{2}\right) \frac{x^{2}}{2}+\left(b_{4}+\cdots\right) \frac{x^{6}}{6}+\cdots,
\end{aligned}
$$

whence we recover $b_{2}, b_{3}, b_{4}$ (in this order) from $y_{2}(g)$, the numbers $a_{i}$ being already known.

Next,

$$
\begin{aligned}
y_{3}(g) & =\Delta_{123}(g)+\Delta_{124}(g) x+\left(\Delta_{134}(g)+\Delta_{125}(g)\right) \frac{x^{2}}{2}+\cdots \\
& =1+c_{3} x+\left(c_{4}+b_{2} c_{3}-b_{3}\right) \frac{x^{2}}{2}+\cdots,
\end{aligned}
$$

whence we recover $c_{3}, c_{4}$ (in this order) from $y_{3}(g)$.

Finally

$$
y_{4}(g)=\Delta_{1234}(g)+\Delta_{1235}(g) x+\cdots=1+d_{4} x+\cdots,
$$

whence $d_{4}$ from $y_{4}(g)$.

Triangular structure on the map $\mathfrak{W}_{N}$. We can express the above as follows. Let $\mathcal{B}:=\mathfrak{W}(N)$, so that

$$
\mathfrak{W}_{N}: N \longrightarrow \mathcal{B} \text {. }
$$

Obviously $N \cong \mathbf{k}^{n(n-1) / 2}$; we define $n(n-1) / 2$ coordinates in $N$ as the elements of a matrix $g \in N$ in the lexicographic order, i.e., $n-1$ elements from the first row (from left to right), $n-2$ elements from the second row, etc.

Let

$$
\mathfrak{W}(g)=\left(y_{1}(g), \ldots, y_{n-1}(g)\right) ;
$$

we define the coordinates of a vector $\mathfrak{W}(g)$ similarly, by taking $n-1$ coefficients of $y_{1}(g)$, then the first $n-2$ coefficients of $y_{2}(g)$, etc. 
Claim 3.9. The above rule defines a global coordinate system on $\mathcal{B}$, i.e., an isomorphism

$$
\mathcal{B} \cong \mathbf{k}^{n(n-1) / 2},
$$

and the matrix of $\mathfrak{W}_{N}$ with respect to the above two lexicographic coordinate systems is triangular with 1 's on the diagonal.

Corollary 3.10. The map $\mathfrak{W}(1.1)$ is an embedding.

Indeed, $X$ is a union of open Schubert cells, the map $\mathfrak{W}$ is $\mathrm{GL}_{n}$-equivariant, and each open cell may be transfered to $N$ using an appropriate $g \in \mathrm{GL}_{n} \cdot{ }^{1}$

\section{Wronskians and tau-functions}

\subsection{Minors of the unit Wronskian}

Consider a $n \times n$ Wronskian matrix

$$
\mathcal{W}_{n}(x)=\mathcal{W}\left(1, x, x^{2} / 2, \ldots, x^{n-1} /(n-1) !\right) .
$$

Claim 4.1. For each $1 \leq i \leq n$ and $I \in \mathcal{C}_{n}^{i}$ we have

$$
\Delta_{[i], I}\left(\mathcal{W}_{n}(x)\right)=\frac{x^{\mathfrak{l}(I)}}{n(I)}
$$

for some $n(I) \in \mathbb{Z}_{>0}$.

The exact value of $n(I)$ will be given below, see Claim 4.5.

\section{Example 4.2.}

$$
\Delta_{14}\left(\mathcal{W}_{4}(x)\right)=\frac{x^{2}}{2}
$$

\subsection{Schur functions, and embedding of a finite Grassmanian into the semi-infinite one}

Recall one of possible definitions for Schur functions, cf. [11, Section 8]. Let

$$
\nu=\left(\nu_{0}, \nu_{1}, \ldots\right)
$$

be a partition, i.e., $\nu_{i} \in \mathbb{Z}_{\geq 0}, \nu_{i} \geq \nu_{i-1}$ and there exists $r$ such that $\nu_{i}=0$ for $i \geq r$.

A Schur function

$$
s_{\nu}\left(h_{1}, h_{2}, \ldots\right)=\operatorname{det}\left(h_{\nu_{i}-i+j}\right)_{i, j=0}^{r-1},
$$

where the convention is $h_{0}=1, h_{i}=0$ for $i<0$, cf. the Jacobi-Trudi formula [8, Chapter I, equation (3.4)].

\section{Examples 4.3.}

$$
\begin{aligned}
& s_{11}=\left|\begin{array}{cc}
h_{1} & h_{2} \\
1 & h_{1}
\end{array}\right|=h_{1}^{2}-h_{2}, \\
& s_{111}=\left|\begin{array}{ccc}
h_{1} & h_{2} & h_{3} \\
1 & h_{1} & h_{2} \\
0 & 1 & h_{1}
\end{array}\right|=h_{1}^{3}-2 h_{1} h_{2}+h_{3} .
\end{aligned}
$$

\footnotetext{
${ }^{1}$ We owe this remark to A. Kuznetsov.
} 


\subsubsection{Electrons and holes}

Let $\mathcal{C}_{\infty / 2}$ denote the set of subsets

$$
S=\left\{a_{0}, a_{1}, \ldots,\right\} \subset \mathbb{Z}
$$

such that both sets $S \backslash \mathbb{N}, \mathbb{N} \backslash S$ are finite, and there exists $d \in \mathbb{Z}$ such that $a_{i}=i-d$ for $i$ sufficiently large.

The number $d=d(S)$ is called the virtual dimension of $S$.

The elements of $\mathcal{C}_{\infty / 2}$ enumerate the cells of the semi-infinite Grassmanian, cf. [11].

We can imagine such $S$ as the set of boxes numbered by $i \in \mathbb{Z}$, with balls put into the boxes with numbers $a_{0}, a_{1}$, etc.

We can define the virtual dimension also as

$$
d(S)=|S \backslash \mathbb{N}|-|\mathbb{N} \backslash S| .
$$

Set

$$
\mathcal{C}_{\infty / 2}^{d}:=\left\{S \in \mathcal{C}_{\infty / 2} \mid d(S)=d\right\} \subset \mathcal{C}_{\infty / 2}
$$

For the moment we will be interested in $\mathcal{C}_{\infty / 2}^{0}$.

We can get each element of $\mathcal{C}_{\infty / 2}^{0}$ by starting from the vacuum state, or Dirac sea

$$
S_{0}=\{0,1, \ldots\}
$$

where the boxes $0,1, \ldots$ being filled, and then moving some $n$ balls to the left.

Let us assign to

$$
S=\left\{a_{0}, a_{1}, \ldots\right\} \in \mathcal{C}_{\infty / 2}^{0}
$$

a partition $\nu=\nu(S)$ by the rule

$$
\nu_{i}=i-a_{i}
$$

This way we get a bijection between $\mathcal{C}_{\infty / 2}^{0}$ and the set of partitions, cf. [11, Lemma 8.1].

Example 4.4. If

$$
S=\{-2,-1,2,3, \ldots\}
$$

then $\lambda(S)=(22)$.

\subsubsection{From semi-infinite cells to finite ones}

For $n \in \mathbb{N}$ let

$$
\mathcal{C}_{\infty / 2, n}=\left\{S=\left(a_{0}, a_{1}, \ldots\right) \mid a_{0} \geq-n, a_{n}=n\right\} \subset \mathcal{C}_{\infty / 2} ;
$$

note that $a_{n}=n$ implies $a_{m}=m$ for all $m \geq n$.

Let

$$
\mathcal{C}_{\infty / 2, n}^{0}=\mathcal{C}_{\infty / 2, n} \cap \mathcal{C}_{\infty / 2}^{0}
$$

Note that we have a bijection

$$
\mathcal{C}_{\infty / 2, n}^{0} \cong \mathcal{C}_{2 n}^{n}
$$


obvious from the "balls in boxes" picture.

Namely, we have inside $\mathcal{C}_{\infty / 2, n}^{0}$ the minimal state

$$
S_{\min }=\left(a_{i}\right) \quad \text { with } \quad a_{i}=1 \quad \text { for }-n \leq i \leq-1 \text { and for } i \geq n
$$

from which one gets all other states in $\mathcal{C}_{\infty / 2, n}^{0}$ by moving the balls to the right, until we reach the maximal state

$$
S_{\max }=\left(b_{i}\right) \quad \text { with } b_{i}=1 \text { and for } i \geq 0 .
$$

For

$$
I \in \mathcal{C}_{2 n}^{n} \cong \mathcal{C}_{\infty / 2, n}^{0} \subset \mathcal{C}_{\infty / 2}^{0}
$$

we will denote by $\nu(I)$ the corresponding partition.

\subsubsection{Transposed cells}

For $I \in \mathcal{C}_{n}^{i}$ let $I^{t}=I^{\prime} \in \mathcal{C}_{n}^{i}$ denote the "opposite", or transposed cell which in "balls and boxes" picture it is obtained by reading $I$ from right to left. The corresponding partition $\lambda\left(I^{t}\right)=\lambda(I)^{t}$ has the transposed Young diagram.

\subsection{Initial Schur functions and the Wronskian}

Let us introduce new coordinates $t_{1}, t_{2}, \ldots$ related to $h_{j}$ by the formula

$$
e^{\sum_{i=1}^{\infty} t_{i} z^{i}}=1+\sum_{j=1}^{\infty} h_{j} z^{j},
$$

cf. [11, equation (8.4)].

For example

$$
h_{1}=t_{1}, \quad h_{2}=t_{2}+\frac{t_{1}^{2}}{2}
$$

etc.

Let us consider the Schur functions $s_{\nu}$ as functions of $t_{i}$. The first coordinate $x=t_{1}$ is called the space variable, whereas $t_{i}, i \geq 2$, are "the times".

We will be interested in the "initial" Schur functions, the values of $s_{\nu}(t)$ for $t_{2}=t_{3}=\cdots=0$.

By definition,

$$
h_{i}(x, 0,0, \ldots)=\frac{x^{i}}{i !} .
$$

Let us return to the unit Wronskian. It is convenient to consider a limit $\mathbb{Z}_{>0} \times \mathbb{Z}_{>0}$ Wronskian matrix

$$
\mathcal{W}_{\infty}(x)=\lim _{n \rightarrow \infty} \mathcal{W}_{n}(x)=\mathcal{W}\left(1, x, x^{2} / 2, \ldots\right)=\left(\begin{array}{ccccc}
1 & x & x^{2} / 2 & x^{3} / 6 & \ldots \\
0 & 1 & x & x^{2} / 2 & \ldots \\
0 & 0 & 1 & x & \ldots \\
\ldots & \ldots & \ldots & \ldots & \ldots
\end{array}\right)
$$

Let

$\nu: \quad \nu_{0} \geq \cdots \geq \nu_{r}>0$ 
be a partition, $S=S(\nu)=\left(a_{i}\right) \in \mathcal{C}_{\infty / 2}^{0}$; define

$$
\ell(S)=\sum_{i \geq 0}\left(i-a_{i}\right)=\sum_{i \geq 0} \nu_{i},
$$

cf. [11, a formula after Proposition 2.6], and "the hook factor"

$$
\mathfrak{h}(S)=\mathfrak{h}(\nu)=\frac{\prod_{0 \leq i<j \leq r}\left(a_{j}-a_{i}\right)}{\prod_{0 \leq i \leq r}\left(r-a_{i}\right) !},
$$

cf. [8, Section I.1, Example 1, equation (4)].

On the other hand suppose that $\nu=\nu(I)$ for some $I=I(\nu) \in \mathcal{C}_{2 n}^{n}$, cf. Claim 3.2.

\section{Claim 4.5.}

(i) $s_{\nu}(x, 0,0, \ldots)=\mathfrak{h}(S) x^{\ell(S)}$,

(ii) $\Delta_{I^{t}}\left(\mathcal{W}_{\infty}(x)\right)=\mathfrak{h}(S) x^{\ell(S)}$.

Proof. (i) is [11, proof of Proposition 8.6]. (ii) is a consequence of a more general Claim 4.12 below.

Example 4.6. Let

$$
I=(1100), \quad S=\{-2,-1,2,3, \ldots\},
$$

then

$$
\nu=(22) ; \quad \ell(S)=4, \quad \mathfrak{h}(S)=\frac{1}{12} .
$$

Then

(i) $s_{\nu}=h_{2}^{2}-h_{1} h_{3}=\frac{t_{1}^{4}}{12}-t_{1} t_{3}+t_{2}^{2}$,

(ii) $I(\nu)=(1100), \quad I^{\prime}(\nu)=(0011)$,

$$
\Delta_{I^{\prime}}\left(\mathcal{W}_{\infty}(x)\right)=\left|\begin{array}{cc}
x^{2} / 2 & x^{3} / 6 \\
x & x^{2} / 2
\end{array}\right|=\frac{x^{4}}{12}
$$

\subsection{Polynomials $y_{n}(g)(x)$ and initial tau-functions: the middle case}

Let $n \geq 1$. For

$$
I \in \mathcal{C}_{2 n}^{n} \cong \mathcal{C}_{\infty / 2, n}^{0} \subset \mathcal{C}_{\infty / 2}^{0}
$$

let $\nu(I)$ denote the corresponding partition.

Consider the Grassmanian

$$
\mathrm{Gr}_{2 n}^{n}=\mathrm{GL}_{2 n} / P_{n, n} .
$$

For a matrix $g \in \mathrm{GL}_{2 n}$ we define its tau-function $\tau(g)$ which will be a function of variables $t_{1}, t_{2}, \ldots$, by

$$
\tau(g)(t)=\tau_{n}(g)=\sum_{I \in \mathcal{C}_{2 n}^{n}} \Delta_{I}(g) s_{\nu(I)}(t),
$$

cf. [11, Proposition 8.3].

Abuse of the notation; better notation: $\tau(\bar{g}), \bar{g} \in \mathrm{Gr}_{2 n}^{n}$. This subspace is described below, see Section 4.6. 
Examples 4.7. (a) $n=1$. There are 2 cells in $\mathrm{Gr}_{2}^{1}=\mathbb{P}^{1}$ :

$(10) \longrightarrow(01)$

(the arrow indicates the Bruhat order), which correspond to the following semi-infinite cells of virtual dimension 0 :

$$
(-1,1,2, \ldots), \quad(0,1,2, \ldots)
$$

which in turn correspond to partitions

$$
(1), \quad()
$$

with Schur functions

$$
s_{(1)}=h_{1}=t_{1}, \quad s_{()}=1 .
$$

Correspondingly, for $g \in \mathrm{GL}_{2}$, the middle tau-function $\tau_{1}(g)$ has 2 summands:

$$
\tau_{1}(g)=\Delta_{1}(g) s_{(1)}+\Delta_{2}(g) s_{()}=a_{11} t_{1}+a_{12}
$$

for $g=\left(a_{i j}\right)$.

Differential equation. Suppose for simplicity that $a=a_{12}=1$, introduce the notation $x=t_{1}$ for the space variable, so $\tau(g)=1+a x$.

Let

$$
u(x)=2 \frac{\mathrm{d}^{2} \log \tau(g)}{\mathrm{d}^{2} x} .
$$

Then

$$
u(x)=-\frac{2 a^{2}}{(1+a x)^{2}} .
$$

It satisfies a differential equation

$$
6 u u_{x}+u_{x x x}=0
$$

which is the stationary $\mathrm{KdV}$.

(b) $n=2$. There are 6 cells in $\mathrm{Gr}_{4}^{2}$ :
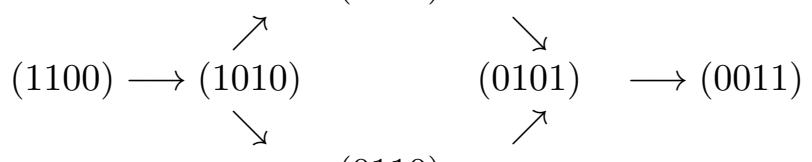

the arrows indicate the Bruhat, or balls in boxes order: we see how 1's (the balls) are moving to the right to the empty boxes.

They correspond to the following semi-infinite cells of virtual dimension 0 :

$$
\begin{aligned}
& (-2,-1,2,3, \ldots), \quad(-2,0,2,3, \ldots), \quad(-2,1,2,3, \ldots), \\
& (-1,0,2,3, \ldots), \quad(-1,1,2,3, \ldots), \quad(0,1,2,3, \ldots),
\end{aligned}
$$


which in turn correspond to partitions

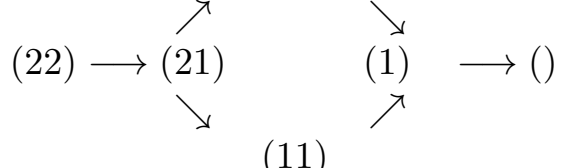

with Schur functions:

$$
\begin{aligned}
& s_{(22)}=h_{2}^{2}-h_{3} h_{1}=\frac{t_{1}^{4}}{12}+t_{2}^{2}-t_{1} t_{3}, \quad s_{(21)}=h_{1} h_{2}-h_{3}=\frac{t_{1}^{3}}{3}-t_{3}, \\
& s_{(2)}=h_{2}=\frac{t_{1}^{2}}{2}+t_{2}, \quad s_{(11)}=h_{1}^{2}-h_{2}=\frac{t_{1}^{2}}{2}-t_{2}, \quad s_{(1)}=h_{1}=t_{1}, \quad s_{()}=1 .
\end{aligned}
$$

Thus for $g \in \mathrm{GL}_{4}, \tau_{2}(g)$ has 6 summands:

$$
\begin{aligned}
\tau_{2}(g)= & \left.\Delta_{12}(g) s_{(22)}+\Delta_{13}(g) s_{(21)}+\Delta_{23}(g) s_{(11)}+\Delta_{14}(g) s_{(2)}+\Delta_{24}(g) s_{(1)}+\Delta_{34}(g) s_{(}\right) \\
= & \Delta_{34}(g)+\Delta_{24}(g) t_{1}+\left(\Delta_{14}(g)+\Delta_{23}(g)\right) \frac{t_{1}^{2}}{2}+\left(\Delta_{14}(g)-\Delta_{23}(g)\right) t_{2} \\
& +\Delta_{13}(g)\left(\frac{t_{1}^{3}}{3}-t_{3}\right)+\Delta_{12}(g)\left(\frac{t_{1}^{4}}{12}+t_{2}^{2}-t_{1} t_{3}\right),
\end{aligned}
$$

where $\Delta_{i j}(g)$ denotes the minor with $i$-th and $j$-th columns. It depends on 3 variables $x=t_{1}$, $y=t_{2}, t=t_{3}$.

We deduce from the above a result from [12, Lemma 7.5]:

\section{Theorem 4.8. If}

$$
\mathfrak{W}(g)=\left(y_{1}(g), \ldots, y_{2 n}(g)\right)
$$

then

$$
\tau(g)(x, 0, \ldots)=\tilde{y}_{n}(g)(x) .
$$

Here we use the reciprocal polynomials $\tilde{y}_{i}(g)$ defined in (3.3).

The initial values of tau-functions $\tau(g)(x, 0, \ldots)$ make their appearance in [11, Proposition 8.6].

Proof. We use the definition (4.2):

$$
\tau_{n}(g)=\sum_{I \in \mathcal{C}_{2 n}^{n}} \Delta_{I}(g) s_{\nu(I)}\left(t_{1}, t_{2}, \ldots\right),
$$

then put $t_{2}=t_{3}=\cdots=0$ in it:

$$
\tau_{n}(g)(x, 0, \ldots)=\sum_{I \in \mathcal{C}_{2 n}^{n}} \Delta_{I}(g) \mathfrak{h}(\nu(I)) x^{\mathfrak{l}(I)}
$$

by Claim 4.5.

On the other hand, by Theorem 3.3

$$
y_{n}(g)(x)=\sum_{I \in \mathcal{C}_{2 n}^{n}} \Delta_{I}(g) m(I) \frac{x^{\mathfrak{l}(I)}}{\mathfrak{l}(I) !},
$$

whence

$$
\tilde{y}_{n}(g)(x)=\sum_{I \in \mathcal{C}_{2 n}^{n}} \Delta_{I}(g) m\left(I^{\prime}\right) \frac{x^{\mathfrak{l}\left(I^{\prime}\right)}}{\mathfrak{l}\left(I^{\prime}\right) !} .
$$


Lemma 4.9 (hook lemma). For all $I$

$$
\mathfrak{h}(\nu(I))=\frac{m\left(I^{\prime}\right)}{\mathfrak{l}\left(I^{\prime}\right) !}
$$

Recall that $m(I)$ is the number of paths from $I_{\min }$ to $I$.

Proof. Induction on the number of cells in the Young diagram of $\nu$.

Example 4.10. $n=4, I=I_{\min }=(1100), I^{\prime}=I_{\max }=(0011), \nu(I)=(22), m\left(I^{\prime}\right)=2$, $\mathfrak{l}\left(I^{\prime}\right)=4$,

$$
S(I)=\{-2,-1,2,3, \ldots\}, \quad \mathfrak{h}(\nu(I))=\frac{1}{12}=2 \frac{1}{24} .
$$

Theorem 4.8 follows from the above.

Example 4.11. Consider $\tau(g)=\tau_{2}(g)$ from Example 4.7(b). Putting $t_{2}=t_{3}=0$ into (4.3) we get

$$
\begin{aligned}
\tau(g)(x, 0,0)= & \Delta_{34}(g)+\Delta_{24}(g) x+\left(\Delta_{14}(g)+\Delta_{23}(g)\right) \frac{x^{2}}{2} \\
& +\Delta_{13}(g) \frac{x^{3}}{3}+\Delta_{12}(g) \frac{x^{4}}{12}=\tilde{y}_{2}(g)(x),
\end{aligned}
$$

see (3.4).

\subsubsection{Stationary solutions}

Let us return to the formula (4.3). We see therefrom that if $g$ is such that

$$
\Delta_{14}(g)=\Delta_{23}(g),
$$

and

$$
\Delta_{13}(g)=\Delta_{12}(g)=0
$$

then $\tau(g)$ does not depend on $t_{2}$ and $t_{3}$, and therefore $\tau(g)=y_{2}(g)$.

Note the Plücker relation

$$
\Delta_{12}(g) \Delta_{34}(g)-\Delta_{13}(g) \Delta_{24}(g)+\Delta_{14}(g) \Delta_{23}(g)=0,
$$

which implies $\Delta_{14}(g)=\Delta_{23}(g)=0$, i.e., the only part which survives will be

$$
\tau(g)\left(t_{1}\right)=\Delta_{34}(g)+\Delta_{24}(g) t_{1} .
$$

\subsection{Case of an arbitrary virtual dimension}

Let $i \leq n$. Consider an embedding

$$
\mathcal{C}_{n}^{i} \hookrightarrow \mathcal{C}_{\infty / 2}^{d(n, i)},
$$

where $d(n, i)$ is chosen in such a way that $\nu\left(I_{\max }\right)=()$ (this defines $d(n, i)$ uniquely). Here $\nu(I)$ denotes the partition corresponding to $I \in \mathcal{C}_{n}^{i}$ under the composition

$$
\mathcal{C}_{n}^{i} \hookrightarrow \mathcal{C}_{\infty / 2}^{d(n, i)} \cong \mathcal{C}_{\infty / 2}^{0},
$$

where the last isomorphism is a shift $\left(a_{j}\right) \mapsto\left(a_{j-i}\right)$, and identifying $\mathcal{C}_{\infty / 2}^{0}$ with the set of partitions. More precisely, for any $d$ a sequence $S=\left(a_{0}, a_{1}, \ldots\right)$ belongs to $\mathcal{C}_{\infty / 2}^{d}$ iff $a_{i}=i-d$ for $i \gg 0$. To such $S$ there corresponds a partition

$$
\lambda(S)=\left(\lambda_{1} \geq \lambda_{2} \geq \cdots\right), \quad \lambda_{i}=a_{i}-i+d .
$$




\subsubsection{Schur functions and minors}

Claim 4.12. Consider an upper triangular Toeplitz matrix

$$
T=\left(\begin{array}{cccc}
1 & h_{1} & h_{2} & \ldots \\
0 & 1 & h_{1} & \ldots \\
0 & 0 & 1 & \ldots \\
\ldots & \ldots & \ldots & \ldots
\end{array}\right)
$$

Let $I \in \mathcal{C}_{n}^{i}$. Then

$$
s_{\nu(I)}(h)=\Delta_{I^{t}}(T),
$$

where $I^{t} \in \mathcal{C}_{n}^{i}$ is the transposed cell (see Section 4.2.3). Note that for any $i$ and any $I \in \mathcal{C}_{n}^{i}$ the function $s_{\nu(I)}(h)$ depends exactly on $h_{1}, \ldots, h_{n-1}$.

Examples 4.13. (a) $n=4, i=2, I=(1100), I^{t}=(0011)$, The corresponding semi-infinite cell is $S(I)=\{-2,-1,2,3, \ldots\}, d(4,2)=0, \nu(I)=(22)$.

(b) $n=4, i=3, I=(1110), I^{t}=(0111)$ The corresponding semi-infinite cell is $S(I)=$ $\{0,1,2,4,5, \ldots\}, d(4,3)=-1, \nu(I)=(111)$.

Afterwards we can apply the same construction as above: to $g \in \mathrm{GL}_{2 n+i}$ we assign a taufunction

$$
\tau(g)(t)=\sum_{I \in \mathcal{C}_{2 n+i}^{n}} \Delta_{I}(g) s_{\nu(I)}(t) .
$$

We deduce from the above a result from [12, Lemma 7.5]:

\section{Theorem 4.14. Let}

$$
\mathfrak{W}(g)=\left(y_{1}(g), \ldots, y_{2 n+i}(g)\right),
$$

then

$$
\tilde{y}_{n}(g)(x)=\tau(g)(x, 0, \ldots) .
$$

Here $\tilde{y}_{n}$ denotes the reciprocal polynomial, see (3.3).

Example 4.15 (projective spaces). Let $n \geq 1$ be arbitrary.

(a) Let $i=1$. There are $n$ cells in $\mathbb{P}^{n-1}=\mathrm{Gr}_{n}^{1}$ :

$$
(10 \ldots 0) \longrightarrow(01 \ldots 0) \longrightarrow \cdots(00 \ldots 1)
$$

which correspond to the semi-infinite cells of virtual dimension $n-1$ :

$$
(10 \ldots 011 \ldots) \longrightarrow(01 \ldots 011 \ldots) \longrightarrow \cdots(00 \ldots 111 \ldots),
$$

(in $\mathrm{BB}$ picture), or

$$
(0, n, n+1, \ldots) \longrightarrow(1, n, n+1, \ldots) \longrightarrow(n-1, n, n+1, \ldots)
$$

which correspond to the partitions

$$
(n-1) \longrightarrow(n-2) \longrightarrow \cdots \longrightarrow()
$$


with Schur functions

$$
s_{(i)}(h)=h_{i},
$$

whence

$$
s_{(i)}(x, 0, \ldots)=\frac{x^{i}}{i !}
$$

cf. (4.1).

So for a matrix $g=\left(a_{i j}\right) \in \mathrm{GL}_{n}$ its first tau-function

$$
\tau_{1}(g)\left(t_{1}, \ldots, t_{n-1}\right)=\sum_{j=0}^{n-1} a_{1, j+1} h_{n-j-1},
$$

and

$$
\tau_{1}(g)(x, 0, \ldots)=\sum_{j=0}^{n-1} a_{1, n-j} \frac{x^{j}}{j !}=\tilde{y}_{1}(g),
$$

as it should be, the contraction map Plücker $\longrightarrow$ Wronsky being the identity.

(b) The dual (conjugate) space $\left(\mathbb{P}^{n-1}\right)^{\vee}$. Let $i=n-1$. There are $n$ cells in $\left(\mathbb{P}^{n-1}\right)^{\vee}=\operatorname{Gr}_{n}^{n-1}$ :

$$
(11 \ldots 110) \longrightarrow \cdots \longrightarrow(101 \ldots 1) \longrightarrow(01 \ldots 1)
$$

which correspond to the semi-infinite cells of virtual dimension -1 :

$$
(11 \ldots 1011 \ldots) \longrightarrow \cdots \longrightarrow(101111 \ldots) \longrightarrow(011 \ldots)
$$

(in BB picture), or to sequences $S$

$$
(0,1, \ldots, n-1, n+1, \ldots) \longrightarrow \cdots \longrightarrow(0,1,3,4, \ldots) \longrightarrow(0,2,3, \ldots),
$$

which correspond to the partitions

$$
\left(1^{n-1}\right) \longrightarrow \cdots \longrightarrow(1) \longrightarrow()
$$

with Schur functions

$$
s_{\left(1^{i}\right)}=e_{i}
$$

(an elementary symmetric function; see [8, Chapter I, equation (3.8)] for the relation between Schur functions of the conjugate partitions). Whence

$$
s_{\left(1^{i}\right)}(x, 0, \ldots)=\frac{x^{i}}{i !} .
$$

For a matrix $g=\left(a_{i j}\right) \in \mathrm{GL}_{n}$ its $(n-1)$-th tau-function

$$
\tau_{n-1}(g)\left(t_{1}, \ldots, t_{n-1}\right)=\sum_{j=1}^{n} \Delta_{[1 \ldots n-j \ldots n]}(g) e_{j} \frac{x^{j-1}}{(j-1) !}
$$

(the coefficients being $(n-1) \times(n-1)$-minors $)$, so

$$
\tau_{n-1}(g)(x, 0, \ldots)=\sum_{j=1}^{n} \Delta_{[1 \ldots n-j \ldots n]}(g) \frac{x^{j-1}}{(j-1) !}=\tilde{y}_{n-1}(g) .
$$




\subsection{Wronskians as tau-functions}

One can express the above as follows. Consider a Tate vector space of Laurent power series

$$
H=\mathbf{k}((z))=\left\{\sum_{i \geq j} a_{i} z^{i} \mid j \in \mathbb{Z}, a_{i} \in \mathbf{k}\right\} .
$$

It is equipped with two subspaces, $H_{+}=\mathbf{k}[[z]]$ and

$$
H_{-}=\left\{\sum_{-j \leq i \leq 0} a_{i} z^{i}, j \in \mathbb{N}\right\} .
$$

Let $\mathrm{Gr}=\mathrm{Gr}_{\infty}^{\infty / 2}$ denote the Grassmanian of subspaces $L \subset H$ of the form

$$
L=L_{0}+z^{k} H_{+}, \quad k \in \mathbb{N},
$$

where $L_{0}=\left\langle f_{1}, \ldots, f_{q}\right\rangle$ is generated by a finite number of Laurent polynomials $f_{i}(z) \in \mathbf{k}\left[z, z^{-1}\right]$, cf. [11, Section 8].

In other words, such $L$ should admit a topological base of the form

$$
\left\{f_{1}(z), \ldots, f_{q}(z), z^{k}, z^{k+1}, \ldots \mid f_{i}(z) \in \mathbf{k}\left[z, z^{-1}\right]\right\} .
$$

For example, in Section 3.4.1 above we see a description of embeddings

$$
\mathbb{P}^{n-1}=\operatorname{Gr}_{n}^{1} \hookrightarrow \mathrm{Gr}, \quad\left(\mathbb{P}^{n-1}\right)^{\vee}=\mathrm{Gr}_{n}^{n-1} \hookrightarrow \mathrm{Gr} .
$$

To each $L \in \mathrm{Gr}$ there corresponds a tau-function

$$
\tau_{L}(t)=\tau_{L}\left(t_{1}, t_{2}, \ldots\right),
$$

cf. [11, Proposition 8.3].

Given a sequence of polynomials

$$
\mathfrak{f}=\left(f_{1}(z), \ldots, f_{m}(z)\right) \subset \mathbf{k}[[z]]_{\leq d}
$$

of degree $\leq d, m \leq d$, let us associate to it a subspace

$$
L(\mathfrak{f})=\left\langle f_{1}\left(z^{-1}\right), \ldots, f_{m}\left(z^{-1}\right), z, z^{2}, \ldots\right\rangle \subset H
$$

belonging to Gr.

\section{Theorem 4.16.}

$$
\tau_{L(\mathfrak{f})}(z, 0, \ldots)=W(\mathfrak{f}) .
$$

This statement is a reformulation of Theorems 4.8 and 4.14 .

\subsection{Full flags and MKP}

For $n \in \mathbb{Z}_{\geq 2}$ define a semi-infinite flag space $\mathcal{F} \ell_{n}^{\infty / 2}$ whose elements are sequences of subspaces

$$
L_{1} \subset \cdots \subset L_{n-1} \subset H=\mathbf{k}((z)), \quad L_{i} \in \mathrm{Gr} \subset \mathrm{Gr}_{\infty}^{\infty} / 2, \quad \operatorname{dim} L_{i} / L_{i-1}=1 .
$$

This is a subspace of the semi-infinite flag space considered in [7, Section 8], whose elements parametrize the rational solutions of the modified Kadomtsev-Petviashvili hierarchy. 
To each flag $F=\left(L_{1} \subset \cdots \subset L_{n-1}\right) \in \mathcal{F} \ell_{n}^{\infty / 2}$ we assign its tau-function which is by definition a collection of $n-1$ grassmanian tau-functions:

$$
\tau_{F}(t):=\left(\tau_{L_{1}}(t), \ldots, \tau_{L_{n-1}}(t)\right), \quad t=\left(t_{1}, t_{2}, \ldots\right) .
$$

Let $g=\left(a_{i j}\right) \in \mathrm{GL}_{n}(\mathbf{k})$, we associate to it as above $n$ polynomials

$$
b_{i}(z)=\sum_{j=0}^{n-1} a_{i, j+1} z^{j}, \quad 1 \leq i \leq n .
$$

We denote

$$
L_{i}(g):=L\left(b_{1}(z), \ldots, b_{i}(z)\right) \in \mathrm{Gr} .
$$

Taking all $1 \leq i \leq n-1$ we get a flag

$$
F(g)=\left(L_{1}(g) \subset \cdots \subset L_{n-1}(g)\right) \in \mathcal{F} \ell_{n}^{\infty / 2}
$$

and can consider the corresponding tau-function

$$
\tau_{F(g)}(t)=\left(\tau_{L_{1}(g)}(t), \ldots, \tau_{L_{n-1}(g)}(t)\right) .
$$

Claim 4.17. For $g \in \mathrm{GL}_{n}(\mathbf{k})$ consider its image under the Wronskian map

$$
\mathfrak{W}(g)=\left(y_{1}(g)(x), \ldots, y_{n}(g)(x)\right) .
$$

Then

$$
\tilde{\mathfrak{W}}(g):=\left(\tilde{y}_{1}(g)(x), \ldots, \tilde{y}_{n}(g)(x)\right)
$$

coincides with the initial value $\tau_{F(g)}(x, 0, \ldots)$ of the tau-function $\tau_{F(g)}$ of the MKP hierarchy corresponding to the semi-infinite flag $F(g)$.

This is an immediate consequence of the Grassmanian case.

Example 4.18. Let $n=4$. Given $g=\left(a_{i j}\right) \in \mathrm{GL}_{4}(\mathbf{k})$, we associate to it four Laurent polynomials encoding its rows

$$
b_{i}(z)=a_{i 1} z^{-2}+\cdots+a_{i 4} z, \quad 1 \leq i \leq 4,
$$

and a semi-infinite flag

$$
F(g)=\left(L_{1}(g) \subset L_{2}(g) \subset L_{3}(g)\right) \in \mathcal{F} \ell_{4}^{\infty / 2},
$$

where

$$
\begin{aligned}
& L_{1}(g)=\left\langle g_{1}(z)\right\rangle+z^{2} H_{+}, \quad L_{2}(g)=\left\langle g_{1}(z), g_{2}(z)\right\rangle+z^{2} H_{+}, \\
& L_{3}(g)=\left\langle g_{1}(z), g_{2}(z), g_{3}(z)\right\rangle+z^{2} H_{+} .
\end{aligned}
$$

We have

$$
\begin{aligned}
& \tau_{1}(g)\left(t_{1}, t_{2}, t_{3}\right)=\sum_{j=0}^{3} a_{1, j+1} h_{3-j}=a_{14}+a_{13} t_{1}+a_{12}\left(\frac{t_{1}^{2}}{2}+t_{2}\right)+a_{11}\left(\frac{t_{1}^{3}}{6}+t_{1} t_{2}+t_{3}\right), \\
& \tilde{y}_{1}(g)(x)=\sum_{j=0}^{3} a_{1,4-j} \frac{x^{j}}{j !},
\end{aligned}
$$


see Example 4.15(a);

$$
\begin{gathered}
\tau_{2}(g)\left(t_{1}, t_{2}, t_{3}\right)=\Delta_{34}(g)+\Delta_{24}(g) t_{1}+\left(\Delta_{14}(g)+\Delta_{23}(g)\right) \frac{t_{1}^{2}}{2}+\left(\Delta_{14}(g)-\Delta_{23}(g)\right) t_{2} \\
+\Delta_{13}(g)\left(\frac{t_{1}^{3}}{3}-t_{3}\right)+\Delta_{12}(g)\left(\frac{t_{1}^{4}}{12}+t_{2}^{2}-t_{1} t_{3}\right), \\
\tilde{y}_{2}(g)(x)=\Delta_{34}(g)+\Delta_{24}(g) x+\left(\Delta_{14}(g)+\Delta_{23}(g)\right) \frac{x^{2}}{2}+\Delta_{13}(g) \frac{x^{3}}{3}+\Delta_{12}(g) \frac{x^{4}}{12},
\end{gathered}
$$

see $(4.3),(4.4)$

$$
\begin{aligned}
& \tau_{3}(g)\left(t_{1}, t_{2}, t_{3}\right)=\Delta_{123}(g)+\Delta_{124}(g) t_{1}+\Delta_{134}(g)\left(\frac{t_{1}^{2}}{2}-t_{2}\right)+\Delta_{234}(g)\left(\frac{t_{1}^{3}}{6}-t_{1} t_{2}+t_{3}\right), \\
& \tilde{y}_{3}(g)(x)=\Delta_{123}(g)+\Delta_{124}(g) x+\Delta_{134}(g) \frac{x^{2}}{2}+\Delta_{234}(g) \frac{x^{3}}{6},
\end{aligned}
$$

see Example 4.15(b).

\section{$5 \quad W 5$ and Desnanot-Jacobi identities}

\section{$5.1 W 5$ identity}

Recall a formula from [10, Section 2.1]. Let

$$
\mathfrak{f}=\left(f_{1}(x), f_{2}(x), \ldots\right)
$$

be a sequence of functions. For a totally ordered finite subset

$$
A=\left\{i_{1}, \ldots, i_{a}\right\} \subset \mathbb{Z}_{>0}
$$

we denote

$$
\mathfrak{f}_{A}=\left(f_{i_{1}}, \ldots, f_{i_{a}}\right), \quad W(A):=W\left(\mathfrak{f}_{A}\right) .
$$

We write $[a]=\{1, \ldots, a\}$.

Lemma 5.1. Let

$$
A=[a+1], \quad B=[a] \cup\{a+2\} .
$$

Then

$$
W(W(A), W(B))=W(A \cap B) \cdot W(A \cup B) .
$$

This is a particular case of $[9$, Section 9$]$.

\section{Example 5.2.}

$$
W\left(W\left(f_{1}, f_{2}\right), W\left(f_{1}, f_{3}\right)\right)=f_{1} W\left(f_{1}, f_{2}, f_{3}\right) .
$$

\subsection{Desnanot-Jacobi, aka Lewis Carrol}

Let $A$ be an $n \times n$ matrix. Denote by $A_{1 n, 1 n}$ the $(n-2) \times(n-2)$ submatrix obtained from $A$ by deleting the first and the $n$-th row and the first and the $n$-th column, etc. Then

$$
\operatorname{det} A \operatorname{det} A_{1 n, 1 n}=\operatorname{det} A_{1,1} \operatorname{det} A_{n, n}-\operatorname{det} A_{1, n} \operatorname{det} A_{n, 1} \text {. }
$$

Let us rewrite (5.1) in the form

$$
W(A \cap B) \cdot W(A \cup B)=W(A) W(B)^{\prime}-W(A)^{\prime} W(B) ;
$$

we see that it resembles (5.2). And indeed, if we apply (5.2) to the Wronskian matrix $\mathcal{W}(A \cup B)$, we get (5.1). This remark appears in [3, the beginning of Section 3]. We leave the details to the reader. 


\subsection{Example: Lusztig vs Wronskian mutations}

Recall the situation (1.1), where we suppose that $m=n$. The main result of [10] describes the compatibility of the map $\mathfrak{W}$ with multiplication by an upper triangular matrix

$$
e_{i, i+1}(c)=I_{n}+c e_{i j}, \quad e_{i j}=\left(\delta_{p i} \delta_{q j}\right)_{p, q} \in \mathrm{GL}_{n}(\mathbf{k}) .
$$

Let $M \in \mathrm{GL}_{n}(\mathbf{k})$,

$$
\begin{aligned}
& \mathfrak{b}(M)=\left(b_{1}(M)(x), b_{2}(M)(x), b_{3}(M)(x), \ldots\right), \\
& \mathfrak{W}(M)=\left(y_{1}(M)(x), y_{2}(M)(x), y_{3}(M)(x), \ldots\right) .
\end{aligned}
$$

The following example is taken from [10, proof of Theorem 4.4]. We have

$$
\mathfrak{W}\left(e_{23} M\right)=\left(y_{1}\left(e_{23} M\right), y_{2}\left(e_{23} M\right), \ldots\right),
$$

where

$$
\mathfrak{b}\left(e_{23} M\right)=\left(b_{1}(M), b_{2}(M)+b_{3}(M), \ldots\right) .
$$

Thus

$$
y_{1}\left(e_{23}(c) M\right)=y_{1}(M),
$$

whereas

$$
y_{2}\left(e_{23}(c) M\right)=y_{2}(M)+c W\left(b_{1}(M), b_{3}(M)\right) .
$$

The $W 5$ identity implies

$$
\begin{aligned}
W\left(y_{2}(M), y_{2}\left(e_{23}(c) M\right)\right) & =c W\left(y_{2}(M), W\left(b_{1}(M), b_{3}(M)\right)\right) \\
& =c W\left(W\left(b_{1}(M), b_{2}(M), W\left(b_{1}(M), b_{3}(M)\right)\right)\right. \\
& =c b_{1} W\left(b_{1}(M), b_{2}(M), b_{3}(M)\right)=c y_{1}(M) y_{3}(M) .
\end{aligned}
$$

The resulting equation

$$
W\left(y_{2}(M), y_{2}\left(e_{23}(c) M\right)\right)=c y_{1}(M) y_{3}(M)
$$

is called a Wronskian mutation equation. It is regarded in [10] as a differential equation of the first order on an unknown function $\tilde{y}_{2}(c, M)=y_{2}\left(e_{23}(c) M\right)$; together with some initial conditions it determines the polynomial $\tilde{y}_{2}(c, M)$ uniquely from given $y_{i}(M), 1 \leq i \leq 3$.

This equation is a modification of a similar differential equation from [9] whose solution is a function

$$
\tilde{y}_{2}^{\prime}(c, M)=y_{2}\left(e_{23}^{\prime}(c) M\right)
$$

where

$$
e_{23}^{\prime}(c)=c e_{23}\left(c^{-1}\right)=c I_{n}+e_{23} .
$$

\section{Acknowledgements}

We are grateful to A. Kuznetsov for a useful discussion, to V. Kac for sending us the paper [7], and to anonymous referees for correcting some inaccuracies and drawing our attention to [4]. V.G. has been partially supported by the HSE University Basic Research Program, Russian Academic Excellence Project 5-100, and by the RSF Grant No. 20-61-46005. 


\section{References}

[1] Benkart G., Meinel J., The center of the affine nilTemperley-Lieb algebra, Math. Z. 284 (2016), 413-439, arXiv:1505.02544.

[2] Berenstein A., Fomin S., Zelevinsky A., Parametrizations of canonical bases and totally positive matrices, Adv. Math. 122 (1996), 49-149.

[3] Crum M.M., Associated Sturm-Liouville systems, Quart. J. Math. Oxford Ser. (2) 6 (1955), $121-127$.

[4] Eremenko A., Gabrielov A., Degrees of real Wronski maps, Discrete Comput. Geom. 28 (2002), 331-347, arXiv:math.AG/0108133.

[5] Fomin S., Zelevinsky A., Recognizing Schubert cells, J. Algebraic Combin. 12 (2000), 37-57, arXiv:math.CO/9807079.

[6] Goldberg L.R., Catalan numbers and branched coverings by the Riemann sphere, Adv. Math. 85 (1991), 129-144.

[7] Kac V.G., Peterson D.H., Lectures on the infinite wedge-representation and the MKP hierarchy, unpublished.

[8] Macdonald I.G., Symmetric functions and Hall polynomials, 2nd ed., Oxford Mathematical Monographs, The Clarendon Press, Oxford University Press, New York, 1995.

[9] Mukhin E., Varchenko A., Critical points of master functions and flag varieties, Commun. Contemp. Math. 6 (2004), 111-163, arXiv:math.AG/0209017.

[10] Schechtman V., Varchenko A., Positive populations, J. Singul. 20 (2020), 342-370, arXiv:1912.11895.

[11] Segal G., Wilson G., Loop groups and equations of KdV type, Inst. Hautes Études Sci. Publ. Math. 61 (1985), 5-65.

[12] Varchenko A., Wright D., Critical points of master functions and integrable hierarchies, Adv. Math. 263 (2014), 178-229, arXiv:1207.2274. 